\title{
Higher derivatives of holomorphic function with positive real part
}

\author{
Shinji YAMASHITA
}

(Received September 9, 1998)

\begin{abstract}
Upper estimates of $\left|f^{(n)}(z)\right| / \operatorname{Re} f(z), n \geq 2$, of $f$ holomorphic and $\operatorname{Re} f>0$ in a plane domain are proposed; the equality conditions are considered in detail.

Key words: function with positive real part, Schwarz-Pick's lemma, hyperbolic domain, Poincaré density, radius of univalency.
\end{abstract}

\section{Introduction}

Let $\mathcal{P}(\Omega)$ be the family of functions $f$ holomorphic with positive real part $\operatorname{Re} f>0$ in a domain $\Omega$ in the complex plane $\mathbf{C}=\{z ;|z|<+\infty\}$. We shall prove some sharp upper estimates of the quotient $\left|f^{(n)}(z)\right| / \operatorname{Re} f(z)$ for $f \in \mathcal{P}(\Omega)$ at $z \in \Omega$ for $n \geq 2$, together with the detailed equality conditions.

The specified case $n=1$ and $\Omega=D \equiv\{z ;|z|<1\}$ is well known. For $f \in \mathcal{P}(D)$,

$$
\frac{\left|f^{\prime}(z)\right|}{\operatorname{Re} f(z)} \leq \frac{2}{1-|z|^{2}}
$$

at each $z \in D$. The extremal functions are essentially $\ell_{\alpha}(z)=(1+\alpha z) /(1-$ $\alpha z)$, where $\alpha \in \partial D \equiv\{z ;|z|=1\}$. More precisely, if the equality holds in (1.1) at a point $z \in D$, then

$$
f(w) \equiv \frac{1-\bar{a} w+\beta(w-a)}{1-\bar{a} w-\beta(w-a)}
$$

where $\beta \in \partial D$ and $a \in D$, so that the equality holds in (1.1) everywhere in $D$; one can prove that $f=A \ell_{\gamma}+i B$, where

$$
A=\frac{1-|a|^{2}}{|1+a \beta|^{2}}>0, \quad B=\frac{-2 \operatorname{Im}(a \beta)}{|1+a \beta|^{2}}, \quad \text { and } \gamma=\frac{\beta+\bar{a}}{1+a \beta} \in \partial D .
$$

See Section 4 for the details on (1.1).

We begin with the case $\Omega=D$ and $n \geq 2$. 
Theorem 1 For $f \in \mathcal{P}(D)$ the estimate

$$
\frac{\left|f^{(n)}(z)\right|}{\operatorname{Re} f(z)} \leq \frac{\ell_{1}^{(n)}(|z|)}{\operatorname{Re} \ell_{1}(|z|)}=\frac{n ! 2}{\left(1-|z|^{2}\right)(1-|z|)^{n-1}}
$$

holds at each point $z \in D$ and for all $n \geq 2$. If the equality holds in (1.2) at a point $z \in D$ and for an $n \geq 2$, then $f=A \ell_{\alpha}+i B$, for an $\alpha \in \partial D$, and for $A>0$ and $B$ both real constants. Conversely, if $f=A \ell_{\alpha}+i B, \alpha \in \partial D$; $A>0$ and $B$ both real constants, then the equality holds in (1.2) at each point of the radius

$$
\mathcal{R}(\alpha)=\{\bar{\alpha} t ; 0 \leq t<1\}
$$

and for each $n \geq 2$, whereas the inequality (1.2) is strict at each point of $D \backslash \mathcal{R}(\alpha)$ and for each $n \geq 2$.

In Section 5 we shall prove Theorem 2 which is proposed in Section 4 and is a version of Theorem 1 for a hyperbolic domain $\Omega$ again with the detailed equality conditions.

\section{Proof of Theorem 1}

We begin with a lemma.

Lemma 1 Let $f$ be holomorphic in $D$, let $0<\rho \leq 1$, and let $A \neq 0$ and $B$ both be complex constants. Set for a fixed $z \in D$,

$$
\frac{f\left(\frac{\rho w+z}{1+\bar{z} \rho w}\right)-B}{A}=\sum_{k=0}^{\infty} b_{k} w^{k}, \quad w \in D .
$$

Then for each $n \geq 1$,

$$
\frac{f^{(n)}(z)}{n !}=\frac{A}{\rho^{n}\left(1-|z|^{2}\right)^{n}} \sum_{k=0}^{n-1}\left(\begin{array}{c}
n-1 \\
k
\end{array}\right)(\bar{z} \rho)^{n-1-k} b_{k+1} .
$$

Proof. Let $g(w)$ be the left-hand side function in (2.1) of the variable $w \in D$. Set

$$
\zeta=\frac{\rho w+z}{1+\bar{z} \rho w}, \quad w \in D, \quad \text { so that } \quad d \zeta=\frac{\rho\left(1-|z|^{2}\right)}{(1+\bar{z} \rho w)^{2}} d w .
$$


Observe that

$$
\frac{(1+\bar{z} \rho w)^{n-1}}{w^{n+1}}=\sum_{k=0}^{n-1}\left(\begin{array}{c}
n-1 \\
k
\end{array}\right)(\bar{z} \rho)^{n-1-k} w^{-k-2} \text { for } w \neq 0
$$

Then

$$
\begin{aligned}
\frac{f^{(n)}(z)}{n !} & =\frac{1}{2 \pi i} \int_{\left|\frac{\zeta-z}{1-\bar{z} \zeta}\right|=\frac{\rho}{2}} \frac{f(\zeta)}{(\zeta-z)^{n+1}} d \zeta \\
& =\frac{A}{\rho^{n}\left(1-|z|^{2}\right)^{n}} \sum_{k=0}^{n-1}\left(\begin{array}{c}
n-1 \\
k
\end{array}\right)(\bar{z} \rho)^{n-1-k} \frac{1}{2 \pi i} \int_{|w|=\frac{1}{2}} \frac{g(w)}{w^{k+2}} d w
\end{aligned}
$$

This is $(2.2)$.

Let $\mathcal{P}_{o}$ be the family of functions $f \in \mathcal{P}(D)$ with $f(0)=1$. A typical member of $\mathcal{P}_{o}$ is $\ell_{\alpha}, \alpha \in \partial D$. For

$$
f(z)=1+\sum_{k=1}^{\infty} a_{k} z^{k}
$$

of $\mathcal{P}_{o}$ we have the estimate $\left|a_{k}\right| \leq 2$ for all $k \geq 1$ and furthermore, $\left|a_{1}\right|=2$ if and only if $f=\ell_{\alpha}$ for an $\alpha \in \partial D$. See [G, p. 80]; the estimate $\left|a_{1}\right| \leq 2$ follows from the Schwarz inequality: $\left|g^{\prime}(0)\right| \leq 1$ for $g=(f-1) /(f+1)$; the equality holds if and only if $g(z) \equiv \alpha z$ for an $\alpha \in \partial D$ or $f=\ell_{\alpha}$.

Simple computation shows that

$$
\ell_{\alpha}\left(\frac{w-b}{1-\bar{b} w}\right) \equiv \frac{1-|b|}{1+|b|} \ell_{\alpha}(w)
$$

in $D$ for all $\alpha \in \partial D$ and all $b \in \mathcal{R}(\alpha)$.

Proof of Theorem 1. Fix $z \in D$ and let

$$
g(w)=\frac{f\left(\frac{w+z}{1+\bar{z} w}\right)-i \operatorname{Im} f(z)}{\operatorname{Re} f(z)}=1+\sum_{k=1}^{\infty} b_{k} w^{k}
$$

We apply Lemma 1 to $g$ with $\rho=1, A=\operatorname{Re} f(z)$, and $B=i \operatorname{Im} f(z)$. Then

$$
\frac{f^{(n)}(z)}{n !}=\frac{\operatorname{Re} f(z)}{\left(1-|z|^{2}\right)^{n}} \sum_{k=0}^{n-1}\left(\begin{array}{c}
n-1 \\
k
\end{array}\right) \bar{z}^{n-1-k} b_{k+1}
$$


which, together with $g \in \mathcal{P}_{o}$, yields that

$$
\frac{\left|f^{(n)}(z)\right|}{n !} \leq \frac{2(1+|z|)^{n-1} \operatorname{Re} f(z)}{\left(1-|z|^{2}\right)^{n}}
$$

this is equivalent to (1.2).

Suppose that the equality holds in (1.2) or in (2.6) at $z$ and for an $n \geq 2$. Then $\left|b_{k+1}\right|=2$ for $0 \leq k \leq n-1$, so that $\left|b_{1}\right|=2$. Hence $g=\ell_{\alpha}$ for an $\alpha \in \partial D$. Since $b_{k+1}=2 \alpha^{k+1}$ for all $k \geq 0$, it follows that

$$
\frac{f^{(m)}(z)}{m !}=\frac{2 \alpha(\bar{z}+\alpha)^{m-1} \operatorname{Re} f(z)}{\left(1-|z|^{2}\right)^{m}}
$$

for all $m \geq 1$. Since the equality holds in (2.6) we then have

$$
|\bar{z}+\alpha|^{n-1}=(1+|z|)^{n-1}
$$

so that $z \in \mathcal{R}(\alpha)$. It then follows from (2.3) for $b=z$ that

$$
f(w)=(\operatorname{Re} f(z)) \ell_{\alpha}\left(\frac{w-z}{1-\bar{z} w}\right)+i \operatorname{Im} f(z)=A \ell_{\alpha}(w)+i B
$$

where

$$
A=\frac{1-|z|}{1+|z|} \operatorname{Re} f(z)>0 \quad \text { and } \quad B=\operatorname{Im} f(z)
$$

Conversely given $f=A \ell_{\alpha}+i B, \alpha \in \partial D, A>0, B$ both real constants, and given $n \geq 2$, we have the chain of identities

$$
\frac{n ! 2}{\left(1-|z|^{2}\right)|1-\alpha z|^{n-1}}=\frac{\left|f^{(n)}(z)\right|}{\operatorname{Re} f(z)}=\frac{n ! 2}{\left(1-|z|^{2}\right)(1-|z|)^{n-1}}
$$

if and only if $z \in \mathcal{R}(\alpha)$.

\section{Application of Theorem 1}

Suppose that $h>0$ is harmonic in $D$. Then we have a holomorphic function $f$ with $\operatorname{Re} f=h$ in $D$. Since

$$
f^{(n)}(z)=2 \frac{\partial^{n} h(z)}{\partial z^{n}}
$$


where

$$
\frac{\partial}{\partial z}=\frac{1}{2}\left(\frac{\partial}{\partial x}-i \frac{\partial}{\partial y}\right)
$$

for $z=x+i y, n \geq 1$, we have

$$
\frac{\left|f^{(n)}\right|}{\operatorname{Re} f}=\frac{2}{h}\left|\frac{\partial^{n} h}{\partial z^{n}}\right| .
$$

We then have the estimate of the right-hand side of (3.1) with the aid of (1.1) and (1.2), together with the detailed equality conditions. Notice that, in the case where $n=1$,

$$
\frac{\left|f^{\prime}\right|}{\operatorname{Re} f}=|\operatorname{grad}(\log h)|
$$

where for $g=\log h$,

$$
|\operatorname{grad} g|=\sqrt{g_{x}^{2}+g_{y}^{2}}
$$

Let $\Gamma$ be the family of $f$ holomorphic in $D$ such that $f(z)+f(w) \neq 0$ for all $z, w \in D$. In particular, $f \in \Gamma$ never vanishes in $D$ and $\mathcal{P}(D) \subset \Gamma$. We call $f \in \Gamma$ a Gel'fer function. In [Y1, Theorem 5, p. 254] we proved that if $f(0)=1$ for $f \in \Gamma$, then $\operatorname{Re} f(z)>0$ in the disk $\{|z|<1 / \sqrt{2}\}$. The constant $1 / \sqrt{2}$ is sharp. For $p=(1+i) / \sqrt{2}$, the function $f(z)=$ $(1-\bar{p} z) /(1+p z)$ is in $\Gamma, f(0)=1$, and further $\operatorname{Re} f(i / \sqrt{2})=0$.

If $f \in \Gamma$ and $z \in D$, then for a constant $\rho, 0<\rho \leq 1$, the function

$$
g(w)=\frac{f\left(\frac{\rho w+z}{1+\bar{z} \rho w}\right)}{f(z)}
$$

of $w \in D$ is in $\Gamma$ with $g(0)=1$. Since $\left|g^{\prime}(0)\right| \leq 2$ (see, for example, [Y1, (G8)]), it follows that

$$
\left|\frac{f^{\prime}(z)}{f(z)}\right| \leq \frac{2}{1-|z|^{2}}
$$

the equality holds for $f=\ell_{\alpha}$ at each point $z=\bar{\alpha} t,-1<t<1$.

We now obtain 
Corollary to Theorem 1 For $f \in \Gamma$ the strict inequality

$$
\left|\frac{f^{(n)}(z)}{f(z)}\right|<\frac{n ! 2 \sqrt{2}(|z|+\sqrt{2})^{n-1}}{\left(1-|z|^{2}\right)^{n}}
$$

holds for all $n \geq 2$ and at each $z \in D$.

Proof. Set $\rho=1 / \sqrt{2}$ and fix $z \in D$. Then the function

$$
g(w)=\frac{f\left(\frac{\rho w+z}{1+\bar{z} \rho w}\right)}{f(z)}=1+\sum_{k=1}^{\infty} b_{k} w^{k}
$$

is in $\mathcal{P}_{o}$. It then follows from (2.2) with $A=f(z)$ that

$$
\left|\frac{f^{(n)}(z)}{f(z)}\right| \leq \frac{n ! 2(1+\rho|z|)^{n-1}}{\rho^{n}\left(1-|z|^{2}\right)^{n}} .
$$

If the equality would hold in (3.3) for an $n \geq 2$, then $\left|b_{k+1}\right|=2$ for $0 \leq k \leq$ $n-1$. Hence $\left|b_{1}\right|=2$, so that there exists $\alpha \in \partial D$ with $g=\ell_{\alpha}$. Then $f$ has $(\rho \bar{\alpha}+z) /(1+\bar{z} \rho \bar{\alpha}) \in D$ as a pole. This is a contradiction.

\section{Hyperbolic domain}

A domain $\Omega$ in the plane $\mathbf{C}$ is called hyperbolic if $\mathbf{C} \backslash \Omega$ contains at least two points. Let $\phi$ be a universal covering projection from $D$ onto a hyperbolic domain $\Omega$ (a projection $\phi: D \rightarrow \Omega$, for short); $\phi$ is holomorphic and $\phi^{\prime}$ is zero-free in $D$. The Poincaré density $\Pi_{\Omega}$ is then the function in $\Omega$ defined by

$$
\Pi_{\Omega}(z)=\frac{1}{\left(1-|w|^{2}\right)\left|\phi^{\prime}(w)\right|}, \quad z \in \Omega
$$

where $z=\phi(w)$; the choice of $\phi$ and $w$ is immaterial as far as $z=\phi(w)$ is satisfied.

Let $\Omega$ and $\Sigma$ both be hyperbolic domains and let $f: \Omega \rightarrow \Sigma$ be holomorphic. The Schwarz-Pick lemma is the estimate of $\left|f^{\prime}\right|$ in terms of $\Pi_{\Omega}$ and $\Pi_{\Sigma}$, namely,

$$
\Pi_{\Sigma}(f(z))\left|f^{\prime}(z)\right| \leq \Pi_{\Omega}(z)
$$

at each point $z \in \Omega$; see, for example, [Y2, p. 304]. If the equality holds at a point $z \in \Omega$, then $f \circ \phi: D \rightarrow \Sigma$ is a projection for each projection $\phi$ : 
$D \rightarrow \Omega$, and, moreover, the equality holds in (4.1) everywhere in $\Omega$. See, for example, [Y2] and [Y3], for recent researches on the Schwarz-Pick lemma.

The case where $\Omega=D$ and $\Sigma$ is the half-plane $H=\{w ; \operatorname{Re} w>0\}$ is of our main interest. Since

$$
\Pi_{D}(z)=\frac{1}{1-|z|^{2}} \quad \text { and } \quad \Pi_{H}(z)=\frac{1}{2 \operatorname{Re} z},
$$

(4.1) for $f \in \mathcal{P}(D)$ is reduced to (1.1).

To consider the higher derivatives we need a device. For a projection $\phi: D \rightarrow \Omega$ we suppose that $z=\phi(w)$. Let $\rho_{\Omega}(z)$ be the greatest $r$ such that $0<r \leq 1$ and $\phi$ is univalent in

$$
\left\{\zeta ;\left|\frac{\zeta-w}{1-\bar{w} \zeta}\right|<r\right\}
$$

which is the non-Euclidean disk of center $w$ and the non-Euclidean radius $\operatorname{arctanh} r$, and also is the disk of

$$
\text { center } \frac{w\left(1-r^{2}\right)}{1-r^{2}|w|^{2}} \in D \quad \text { and radius } \quad \frac{r\left(1-|w|^{2}\right)}{1-r^{2}|w|^{2}} \leq 1 .
$$

Again $\rho_{\Omega}(z)$ is independent of the particular choice of $\phi$ and $w$ as far as $z=\phi(w)$ is satisfied. We may therefore call $\rho_{\Omega}(z)$ the radius of univalency of $\Omega$ at $z \in \Omega$. In particular, the set

$$
\Delta(z)=\left\{\phi(\zeta) ;\left|\frac{\zeta-w}{1-\bar{w} \zeta}\right|<\rho_{\Omega}(z)\right\}, \quad z=\phi(w),
$$

is a simply connected domain depending only on $z \in \Omega ; \Delta(z)$ will be considered in Section 6.

Theorem 2 For $f \in \mathcal{P}(\Omega)$ of a hyperbolic domain $\Omega \subset \mathbf{C}$ the inequality

$$
\frac{\left|f^{(n)}(z)\right|}{\operatorname{Re} f(z)} \leq 2 \cdot \frac{(2 n-1) !}{(n-1) !}\left(\frac{\Pi_{\Omega}(z)}{\rho_{\Omega}(z)}\right)^{n}
$$

holds for each $n \geq 2$ and at each $z \in \Omega$. If the equality holds in (4.3) at a point $z \in \Omega$ and for an $n \geq 2$, then the following two items hold.

(I) There exist complex constants $Q \neq 0$ and $R$ such that $\Omega$ is the slit domain

$$
\Omega=\mathbf{C} \backslash\left\{Q t+R ; t \leq-\frac{1}{4}\right\} ;
$$


in particular, $\rho_{\Omega}(z) \equiv 1$.

(II) The function $f$ is of the form

$$
f(w)=A \sqrt{\frac{Q}{4 w+Q-4 R}}+i B,
$$

where $A>0$ and $B$ are real constants and the branch of $\sqrt{ }$ is chosen so that $f(R)=A+i B$.

Conversely suppose that $f$ of (4.5) is given in $\Omega$ of (4.4). Then the equality holds in (4.3) at each point of the half-line

$$
\mathcal{L}=\left\{Q t+R ; t>-\frac{1}{4}\right\}
$$

and for each $n \geq 2$, whereas the inequality (4.3) is strict at each point of $\Omega \backslash \mathcal{L}$ and for each $n \geq 2$.

The function of (4.5) maps $\Omega$ of (4.4) univalently onto $H$.

The inequality (4.3) in the specified case $\Omega=D$ reads that

$$
\frac{\left|f^{(n)}(z)\right|}{\operatorname{Re} f(z)} \leq 2 \cdot \frac{(2 n-1) !}{(n-1) !} \cdot \frac{1}{\left(1-|z|^{2}\right)^{n}}
$$

at each $z \in D$ and for each $n \geq 2$. Since

$$
2^{n-1}<\frac{(2 n-1) !}{n !(n-1) !} \quad \text { for } \quad n \geq 2,
$$

(4.7) is worse than (1.2). Hence Theorem 2 is not an extension of Theorem 1.

As preparation for the proof of Theorem 2 we begin with the class $\mathcal{S}$ of functions $f$ holomorphic and univalent in $D$ with $f(0)=f^{\prime}(0)-1=0$. Typical members of $\mathcal{S}$ are the rotations of the Koebe function $K=K_{1}$, namely, $K_{\alpha}(z)=z /(1-\alpha z)^{2}, \alpha \in \partial D$. K.S. Chua's coefficient theorem [C, Theorem 2 for the inverse function $f^{*}$ of $f \in \mathcal{S}$ in $f(D)$ is the following. Let $f^{* k}$ be the $k$-th power of $f^{*}(k=1,2, \cdots)$ having the expansion

$$
f^{* k}(w)=\sum_{n=k}^{\infty} B_{n k}(f) w^{n}
$$

in a neighborhood of 0 . Note that $B_{k k}(f)=1$. Then

$$
\left|B_{n k}(f)\right| \leq\left|B_{n k}(K)\right|
$$


for $n \geq k \geq 1$. If $n \geq 2$ and if the equality holds in (4.8) for a pair $n, k$ with $n>k$, then $f=K_{\alpha}$ for an $\alpha \in \partial D$, so that the equality holds in (4.8) for all $n \geq k \geq 1$. Chua observed that [C, (8) and (16)]

$$
B_{n k}(K)=(-1)^{n-k} \frac{k}{n}\left(\begin{array}{c}
2 n \\
n-k
\end{array}\right), \quad 1 \leq k \leq n,
$$

and further that

$$
\sum_{k=1}^{n}\left|B_{n k}(K)\right|=\left(\begin{array}{c}
2 n-1 \\
n
\end{array}\right) .
$$

For later use we remark that

$$
\frac{1-K^{*}(w)}{1+K^{*}(w)}=\frac{1}{\sqrt{4 w+1}}
$$

because

$$
K^{*}(w)=\frac{2 w+1-\sqrt{4 w+1}}{2 w}
$$

for $w \in K(D)$. It follows from

$$
\left(K_{\alpha}\right)^{*}(w)=\bar{\alpha} K^{*}(\alpha w), \quad w \in K_{\alpha}(D),
$$

that

$$
B_{n k}\left(K_{\alpha}\right)=B_{n k}(K) \alpha^{n-k}, \quad \text { for } 1 \leq k \leq n \quad \text { and } \quad \alpha \in \partial D
$$

\section{Proof of Theorem 2}

Supposing first that $0 \in \Omega$ and $\phi(0)=\phi^{\prime}(0)-1=0$ for a projection $\phi: D \rightarrow \Omega$, and further that $f(0)=1$, we shall prove that

$$
\frac{\rho_{\Omega}(0)^{n}\left|f^{(n)}(0)\right|}{n !} \leq 2\left(\begin{array}{c}
2 n-1 \\
n
\end{array}\right)
$$

for all $n \geq 2$. Furthermore we shall observe that if the equality holds in (5.1) for an $n \geq 2$, then there exists $\beta \in \partial D$ such that $\Omega=K_{\beta}(D)$ and

$$
f(w)=\frac{1}{\sqrt{4 \beta w+1}}
$$

for $w \in \Omega$, the branch satisfying $f(0)=1$. 
Set $\rho=\rho_{\Omega}(0)$. Then $\Phi(z)=\rho^{-1} \phi(\rho z)$ is a member of $\mathcal{S}$ and

$$
F(z) \equiv f(\rho \Phi(z))=f(\phi(\rho z))
$$

is of $\mathcal{P}_{o}$. Applying the composite function theorem [T, Theorem 1] to

$$
F \circ \Phi^{*}(\zeta)=f(\rho \zeta), \quad \zeta=\Phi(z) \in \Phi(D),
$$

we have

$$
\rho^{n} f^{(n)}(\rho \zeta)=\left(F \circ \Phi^{*}\right)^{(n)}(\zeta)=\sum_{k=1}^{n} A_{n k}(\zeta) F^{(k)}\left(\Phi^{*}(\zeta)\right)
$$

where

$$
A_{n k}(\zeta)=\frac{1}{k !} \sum_{j=1}^{k}(-1)^{k-j}\left(\begin{array}{l}
k \\
j
\end{array}\right)\left(\Phi^{*}\right)^{k-j}(\zeta)\left(\Phi^{* j}\right)^{(n)}(\zeta)
$$

and further, $\left(\Phi^{*}\right)^{k-j}$ is the $(k-j)$-th power of $\Phi^{*}$ with $\left(\Phi^{*}\right)^{0}=1$ and $\left(\Phi^{* j}\right)^{(n)}$ is the $n$-th derivative of the $j$-th power of $\Phi^{*}, 1 \leq j \leq k \leq n$. Setting $\zeta=0$ in $(5.3)$ one now has

$$
\frac{\rho^{n} f^{(n)}(0)}{n !}=\sum_{k=1}^{n} B_{n k}(\Phi) \frac{F^{(k)}(0)}{k !} .
$$

Since $\left|F^{(k)}(0)\right| \leq k ! 2$ for all $k \geq 1$, and since (4.8) holds for $\Phi \in \mathcal{S}$, one immediately has (5.1) with the aid of (4.9).

Suppose that the equality holds in (5.1) for an $n \geq 2$. Then $\left|B_{21}(\Phi)\right|=$ $\left|B_{21}(K)\right|$ and $\left|F^{\prime}(0)\right|=2$. Hence we have $\alpha$ and $\beta$ of $\partial D$ such that $F=\ell_{\alpha}$ and $\Phi=K_{\beta}$. If $\rho<1$, then $f(\phi(\rho \bar{\alpha}))=\ell_{\alpha}(\bar{\alpha})=\infty$, so that $f$ is not holomorphic in $\Omega$. Hence $\rho=1$, so that

$$
f=F \circ \Phi^{*}=\ell_{\alpha} \circ\left(K_{\beta}\right)^{*}
$$

and $\Omega=\phi(D)=\Phi(D)=K_{\beta}(D)$. To have (5.2) we next show that $\alpha \bar{\beta}=-1$. Set $\gamma=-\alpha \bar{\beta}$. Then (5.4) reads that

$$
\begin{aligned}
\frac{f^{(n)}(0)}{n !} & =\sum_{k=1}^{n} B_{n k}\left(K_{\beta}\right) \frac{\left(\ell_{\alpha}\right)^{(k)}(0)}{k !} \\
& =\sum_{k=1}^{n}(-\beta)^{n-k}\left|B_{n k}(K)\right| 2 \alpha^{k}
\end{aligned}
$$




$$
=2(-\beta)^{n} \sum_{k=1}^{n} \gamma^{k}\left|B_{n k}(K)\right| .
$$

It then follows from (4.9) that

$$
\sum_{k=1}^{n}\left|B_{n k}(K)\right|=\left(\begin{array}{c}
2 n-1 \\
n
\end{array}\right)=\frac{\left|f^{(n)}(0)\right|}{n ! 2}=\left|\sum_{k=1}^{n} \gamma^{k}\right| B_{n k}(K)|| .
$$

Hence, squaring the left- and the right-most terms one has

$$
\sum\left|B_{n k}(K) \| B_{n j}(K)\right|\left(1-\gamma^{k-j}\right)=0,
$$

where the summation is taken over all $k, j$ with $1 \leq k \leq n, 1 \leq j \leq n$; note that $n \geq 2$. Since $\operatorname{Re}\left(1-\gamma^{k-j}\right) \geq 0$, it follows that $\operatorname{Re}\left(1-\gamma^{k-j}\right)=0$ so that $\gamma^{k-j}=1$ for $k \neq j, 1 \leq k \leq n, 1 \leq j \leq n$. Hence $\gamma=1$. We thus have, with the aid of (4.10), that

$$
f(w)=\ell_{\alpha} \circ\left(K_{\beta}\right)^{*}(w)=\frac{1+\alpha \bar{\beta} K^{*}(\beta w)}{1-\alpha \bar{\beta} K^{*}(\beta w)}=\frac{1-K^{*}(\beta w)}{1+K^{*}(\beta w)}=\frac{1}{\sqrt{4 \beta w+1}} .
$$

Given $f$ of $(5.2)$ in $\Omega=K_{\beta}(D), \beta \in \partial D$, we consider the set $E$ of points $z \in \Omega$ where the equality holds in (4.3) for all $n \geq 2$. Since $w=K_{\beta}(\zeta) \in \Omega$, $\zeta \in D$, simple calculation yields that

$$
\operatorname{Re} f(w)=\frac{1-|\zeta|^{2}}{|1+\beta \zeta|^{2}}, \quad\left|f^{(n)}(w)\right|=2 \cdot \frac{(2 n-1) !}{(n-1) !}\left|\frac{1-\beta \zeta}{1+\beta \zeta}\right|^{2 n+1}
$$

because

$$
\prod_{k=0}^{n-1}\left(\frac{1}{2}+k\right)=2^{1-2 n} \cdot \frac{(2 n-1) !}{(n-1) !}
$$

and further, $\rho_{\Omega}(w) \equiv 1$, and

$$
\frac{1}{\Pi_{\Omega}(w)}=\frac{\left(1-|\zeta|^{2}\right)|1+\beta \zeta|}{|1-\beta \zeta|^{3}}
$$

for $\zeta \in D$. Hence

$$
\frac{\left|f^{(n)}(w)\right|}{\Pi_{\Omega}(w)^{n} \operatorname{Re} f(w)}=2 \cdot \frac{(2 n-1) !}{(n-1) !}\left(\frac{1-|\zeta|^{2}}{\left|1-\beta^{2} \zeta^{2}\right|}\right)^{n-1}
$$

for $\zeta \in D$. Consequently, $w=K_{\beta}(\zeta)$ is in $E$ if and only if $\beta \zeta$ is on the real 
diameter $(-1,1)$ or equivalently, if and only if

$$
w \in \Lambda \equiv\left\{\bar{\beta} t ; t>-\frac{1}{4}\right\}
$$

Hence $E=\Lambda$. It is easy to prove that the inequality (4.3) is strict at each point $z \in K_{\beta}(\Omega) \backslash \Lambda$ and for each $n \geq 2$.

To prove (4.3) at $z=a \in \Omega$ we choose a projection $\phi: D \rightarrow \Omega$, with $\phi(0)=a$ and consider the domain

$$
\Sigma=\left\{\frac{\zeta-a}{\phi^{\prime}(0)} ; \zeta \in \Omega\right\}
$$

for which $0 \in \Sigma$ and $\psi=(\phi-a) / \phi^{\prime}(0)$ is a projection $\psi: D \rightarrow \Sigma$ with $\psi(0)=\psi^{\prime}(0)-1=0$. Then the function

$$
g(w)=\frac{f\left(a+\phi^{\prime}(0) w\right)-i \operatorname{Im} f(a)}{\operatorname{Re} f(a)}
$$

is in $\mathcal{P}(\Sigma)$ with $g(0)=1$. Since $g^{(n)}(0)=\phi^{\prime}(0)^{n} f^{(n)}(a) / \operatorname{Re} f(a)$, since $\rho_{\Sigma}(0)=\rho_{\Omega}(a)$, and since $\left|\phi^{\prime}(0)\right|=1 / \Pi_{\Omega}(a)$, we may apply (5.1) to $g$ in $\Sigma$ to have

$$
\left(\frac{\rho_{\Omega}(a)}{\Pi_{\Omega}(a)}\right)^{n} \frac{\left|f^{(n)}(a)\right|}{n ! \operatorname{Re} f(a)}=\frac{\rho_{\Sigma}(0)^{n}\left|g^{(n)}(0)\right|}{n !} \leq 2\left(\begin{array}{c}
2 n-1 \\
n
\end{array}\right)
$$

for all $n \geq 2$. This is equivalent to (4.3) for $z=a$. If the equality holds in (4.3) at $z=a$, thenwe have (I) and (II) with

$$
Q=\bar{\beta} \phi^{\prime}(0), \quad R=a, \quad A=\operatorname{Re} f(a), \quad \text { and } \quad B=f(a) .
$$

The detailed proof is obvious.

Remark 1. How about the case $n=1$ in Theorem 2] Since (4.1) for $\Sigma=H$ is valid, we have

$$
\frac{\left|f^{\prime}(z)\right|}{\operatorname{Re} f(z)} \leq 2 \Pi_{\Omega}(z) \leq 2 \frac{\Pi_{\Omega}(z)}{\rho_{\Omega}(z)} .
$$

Suppose that the left- and the right-most are the same in (5.6). Then $\rho_{\Omega}(z)=1$, so that $\Omega$ must be simply connected, and furthermore, the equalities hold in (5.6) for every poit of $\Omega$. The function $F$ in the proof must be $\ell_{\alpha}$ for some $\alpha \in \partial D$ because $\left|F^{\prime}(0)\right|=2$ (and we have no explicit 
form for $\Phi=\phi$.$) We can also prove (the weaker result)$

$$
\frac{\left|f^{\prime}(z)\right|}{\operatorname{Re} f(z)} \leq 2 \frac{\Pi_{\Omega}(z)}{\rho_{\Omega}(z)}
$$

by the same method as in the proof of Theorem 2 .

Remark 2. It is known that $\mathcal{P}(\Omega)=\mathbf{C}$ if the closed set $\mathbf{C} \backslash \Omega$ is of logarithmic capacity zero. In other words, $\mathcal{P}(\Omega)=\mathbf{C}$ if $\Omega \in O_{H P}=O_{G}$; see [AS, p. 208]. More generally, $g$ is holomorphic and bounded in modulus by $M>0$ in $\Omega$ if and only if $f=(g+M) /(g-M) \in \mathcal{P}(\Omega)$. Hence one observes that $\mathcal{P}(\Omega)=\mathbf{C}$ if and only if $\Omega \in O_{A B}$.

\section{Positive harmonic function in $\Omega$}

Let $h>0$ be harmonic in a hyperbolic domain $\Omega$. Then for each $\Delta(z)$ of (4.2) we have a holomorphic function $f$ with $\operatorname{Re} f=h$ in $\Delta(z)$. Since the proof of Theorem 2 is "local" in its character, we have

$$
\left|\frac{\partial^{n} h(z)}{\partial z^{n}}\right| \frac{1}{h(z)} \leq \frac{(2 n-1) !}{(n-1) !}\left(\frac{\Pi_{\Omega}(z)}{\rho_{\Omega}(z)}\right)^{n}
$$

at each $z \in \Omega$ and for each $n \geq 1$. Actually, (4.3) for the present $f$ is valid, and the case $n=1$ is obvious by $\rho_{\Omega}(z) \leq 1$. If the equality holds at a point $z \in D$, then $\rho_{\Omega}(z)=1$, so that $f$ can be defined in the whole $\Omega$, the slit domain of (4.4). The equality conditions are different according as $n=1$ or $n>1$.

\section{References}

[AS] Ahlfors L.V. and Sario L., Riemann Surfaces. Princeton University Press, Princeton, 1960.

[C] Chua K.S., Derivatives of univalent functions and the hyperbolic metric. Rocky Mt. J. Math. 26 (1996), 63-75.

[G] Goodman A.W., Univalent Functions. Volume I. Mariner Publ. Co., Tampa, Florida, 1983.

[T] Todorov P.G., New explicit formulas for the $\mathrm{n}$ th derivative of composite functions. Pacific J. Math. 92 (1981), 217-236.

[Y1] Yamashita S., Gelfer functions, integral means, bounded mean oscillation, and univalency. Trans. Amer. Math. Soc. 321 (1990), 245-259.

[Y2] Yamashita S., The Pick version of the Schwarz lemma and comparison of the Poincaré densities. Ann. Acad. Sci. Fenn. Ser. A. I. Math. 19 (1994), 291-322. 
[Y3] Yamashita S., Goluzin's extension of the Schwarz-Pick inequality. J. of Inequal. \& Appl. 1 (1997), 345-356.

Department of Mathematics Tokyo Metropolitan University Minami-Osawa, Hachioji Tokyo 192-0397, Japan

E-mail: yamashin@comp.metro-u.ac.jp 